\title{
Noninvasive Ventilation as an Important Adjunct to an Exercise Training Program in Subjects With Moderate to Severe COPD
}

\author{
Kamilla Tays Marrara PhD, Valéria Amorim Pires Di Lorenzo PhD, Rodrigo B Jaenisch PhD, \\ Ramona Cabiddu PhD, Tatiana de Oliveira Sato PhD, Renata Gonçalves Mendes PhD, \\ Claudio R Oliveira PhD, Dirceu Costa PhD, and Audrey Borghi-Silva PhD
}

\begin{abstract}
BACKGROUND: The primary objective of this study was to investigate whether noninvasive ventilation (NIV) can positively affect exercise capacity, maximum oxygen uptake $\left(\dot{\mathrm{V}}_{\mathrm{O}_{2}}\right)$, and symptoms after a 6-week physical training program for subjects with moderate to very severe COPD. METHODS: 47 subjects with COPD who were enrolled in a physical training program were randomized to either physical training alone or NIV + physical training (NIV-Physical training). Physical training consisted of dynamic aerobic exercises on a treadmill 3 times/week for 6 weeks, for a total of 18 sessions. NIV was titrated according to the subject's tolerance at rest and during exercise. Assessments included physiological responses and symptoms at the incremental cardiopulmonary exercise test peak and during submaximal exercise on a treadmill, 6-min walk distance, maximum inspiratory $\left(\mathbf{P}_{I \max }\right)$ and expiratory pressure $\left(\mathbf{P}_{\text {Emax }}\right)$, BODE index, and health-related quality of life. RESULTS: 43 subjects completed the 6-week physical training program. Both groups improved 6-min walk distance, $P_{\text {Imax }}$, BODE index, and quality of life, and no differences were found between groups. However, significant improvements were observed for subjects in the NIVPhysical training group with regard to $P_{\text {Emax }}$, maximum $\dot{\mathrm{V}}_{\mathrm{O}_{2}}$, maximum metabolic equivalents, circulatory power, and maximum $\mathrm{S}_{\mathrm{pO}_{2}}$. CONCLUSIONS: A 6-week physical training program alone can improve tolerance for exercise and quality of life, in addition to reducing the risk of mortality. However, NIV associated with a physical training program was shown to have an additive beneficial effect on powerful prognostic markers (maximum $\dot{\mathrm{V}}_{\mathrm{O}_{2}}$ and circulatory power) and to reduce symptoms and improve oxygen saturation in subjects with moderate to very severe COPD. Key words: noninvasive ventilation; exercise; rehabilitation; physiological adaptations; exercise tolerance; quality of life; chronic obstructive pulmonary disease. [Respir Care 2018;63(11):1388-1398. (C) 2018 Daedalus Enterprises]
\end{abstract}

\section{Introduction}

Patients with COPD usually present exercise intolerance, resulting from changes in lung function, dyspnea,

Drs Marrara, Jaenisch, Cabiddu, Mendes, and Borghi-Silva are affiliated with the Cardiopulmonary Physiotherapy Laboratory, Federal University of São Carlos, São Carlos, São Paulo, Brazil. Dr Marrara is also affiliated with the Central University of São Paulo, São Carlos, São Paulo, Brazil. Dr Di Lorenzo is affiliated with the Research Laboratory in Spirometry and Respiratory Physiotherapy, Federal University of São Carlos, São Carlos, São Paulo, Brazil. Dr Sato is affiliated with the Preventive Physiotherapy and Ergonomics Laboratory, Federal University of São Carlos, São Carlos, São Paulo, Brazil. Dr Oliveira is affiliated with the Department of Medicine, Federal University of São Carlos, São Carlos, São Paulo, Brazil. Dr Costa is affiliated with the University of Nove de Julho, São Paulo, Brazil. and muscle fatigue. ${ }^{1,2}$ Reduced respiratory and peripheral muscle strength and/or endurance have a negative impact on the performance of daily life activities, ${ }^{3}$ which reduces the quality of life of patients with moderate to severe

\footnotetext{
This research was supported by the São Paulo Research Foundation (FAPESP 2009/01842-0). The authors have disclosed no other conflicts of interest.

Correspondence: Audrey Borghi-Silva PhD, Physiotherapy Department, Federal University of São Carlos, Rod. Washington Luis, km 235, SP-310, CEP 13565-905, São Carlos, São Paulo, Brazil. E-mail: audrey@ufscar.br.
}

DOI: $10.4187 /$ respcare. 05763 


\section{Exercise Training With NIV in Subjects With COPD}

COPD. In addition, more severely affected patients show more reduced respiratory and peripheral muscle strength and endurance, as well as more hypoxemia and symptoms of hyperinflation ${ }^{4}$ during exercise than patients with mild COPD. ${ }^{3,5}$

It is already widely accepted that physical training is an essential part of nonpharmacologic treatment in moderate to severe COPD and is considered a key point in pulmonary rehabilitation programs aimed at improving exercise capacity, reducing symptoms, and enhancing quality of life. ${ }^{6,7}$ With physical training being widely recognized and accepted as a fundamental component of a rehabilitation process, exercise intensity is an important factor to considered to achieve satisfactory physiological gains and relevant clinical outcomes. ${ }^{89}$ Many patients with COPD fail to reach a desirable level of intensity and consequently do not experience the expected adaptations. ${ }^{6-9}$ Desaturation during exercise ${ }^{10}$ and the hemodynamic changes caused by dynamic hyperinflation, ${ }^{11}$ severe dyspnea, and lower limb fatigue are the main important factors that affect the ability to maintain exercise intensities sufficient to produce physiological adaptations during a physical training program. ${ }^{6-11}$

Noninvasive ventilation (NIV) has been proposed as a way to unload the respiratory muscles, ${ }^{12}$ reduce symptoms, ${ }^{13}$ and improve exercise tolerance. ${ }^{14}$ The use of NIV in association with a formal physical training program has been shown to be able to unload ventilatory muscles, reduce symptoms, and improve exercise capacity in subjects with COPD. ${ }^{15-20}$ However, the studies that demonstrated these effects have fundamental drawbacks, such as small sample sizes and technical heterogeneity in terms of ventilatory modes, inspiratory levels, disease severity, and physical training intensity. In this field of study, randomized clinical trials with larger sample sizes based on statistical power calculations should also be designed to investigate the effect of training duration and intensity. ${ }^{6,21}$

The primary objective of this study was to investigate whether NIV can positively impact exercise capacity, maximum oxygen uptake $\left(\dot{\mathrm{V}}_{\mathrm{O}_{2}}\right)$, and symptoms after a 6-week physical training program in subjects with moderate to very severe COPD. Our hypothesis is that NIV will positively impact exercise capacity and symptoms in these subjects.

\section{Methods}

\section{Study Design and Participants}

This was a prospective, randomized, controlled trial. Subjects were invited to participate in this study by means of posters and flyers distributed in the city and via Internet postings. In addition, some patients were referred to the respiratory physiotherapy program of our institution. Inclusion criteria were clinical diagnosis of COPD, according to the Global

\section{QUICK LOOK}

\section{Current knowledge}

COPD is a systemic disease characterized by obstruction of airflow and musculoskeletal impairment, which leads to a loss of physical conditioning and dyspnea by means of a vicious cycle in which patients limit their activities to ease symptoms. Noninvasive ventilation (NIV) combined with a formal physical training program can be an effective adjunct to unload ventilatory work, reduce symptoms, and improve exercise capacity in patients with COPD.

\section{What this paper contributes to our knowledge}

The described 6-week treadmill physical training program was beneficial for subjects with moderate to very severe COPD in that it provided an improvement in physical capacity and quality of life. In addition, the combination of the proposed training program with NIV had even greater beneficial effects, as demonstrated by the prognostic markers of COPD.

Initiative for Chronic Obstructive Lung Disease (GOLD) ${ }^{22}$; previous history of smoking ( $>10$ pack-years); ventilatory reserve $<20 \% ; \mathrm{S}_{\mathrm{pO}_{2}}$ ) at rest $>90 \%$ or $\mathrm{S}_{\mathrm{pO}_{2}}$ at maximum exercise $>80 \%$; maximum inspiratory pressure $\left(\mathrm{P}_{\text {Imax }}\right)<70 \%$ of predicted; clinical stability in the last 2 months; no changes in medication dosage for 1 month and no participation in a physical training program for 3 months.

Exclusion criteria were the presence of musculoskeletal, orthopedic, or neurological conditions that would preclude participation in the exercise program; a previous history consistent with heart disease, diabetes mellitus, uncontrolled hypertension, or other concomitant respiratory diseases; and failure to comply with the research protocol or to receive NIV. The study was approved by the ethics committee of our institution and registered in the Clinical Trials Register (NCT01464736).

Of the 186 subjects initially evaluated, 47 were included in this study (Fig. 1). They were randomized to receive physical training alone or physical training with NIV (NIVPhysical training) by sealed envelopes that were opened by a single investigator who did not know the purpose of the study.

\section{Outcome Measurements}

Subjects underwent the study assessments on an outpatient basis, before and after a 6-week physical training program. The primary outcome measurement was maximum $\dot{\mathrm{V}}_{\mathrm{O}_{2}}$, as the main physiological adaptation to training. 


\section{Exercise Training With NIV in Subjects With COPD}

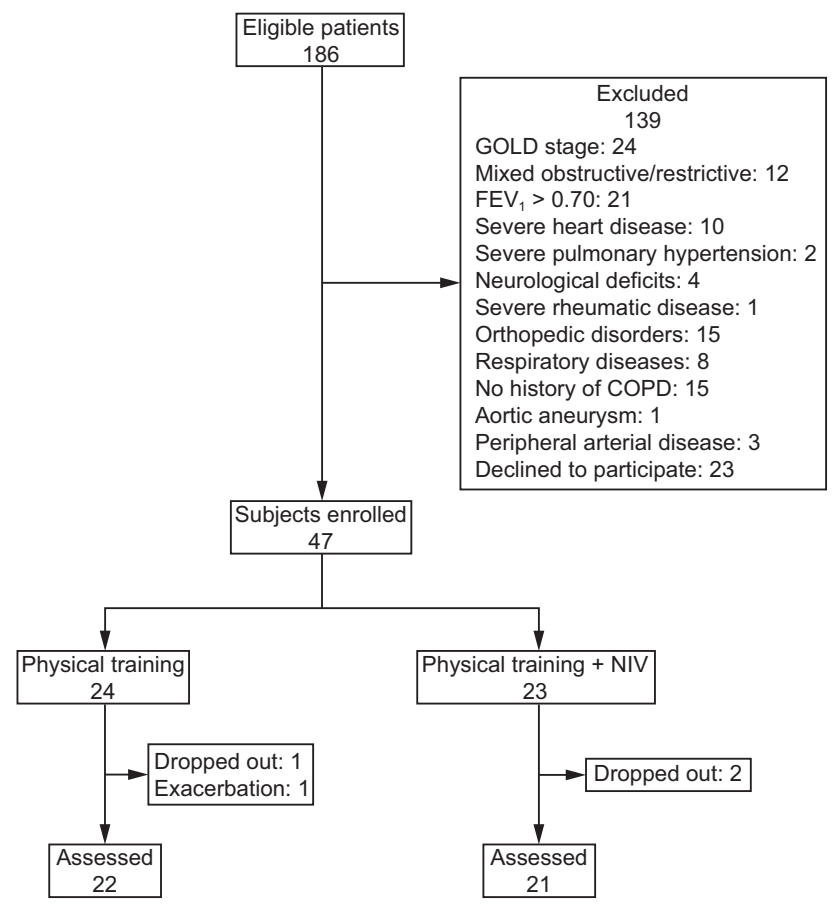

Fig. 1. Flow chart. GOLD = Global Initiative for Chronic Obstructive Lung Disease; CHF = congestive heart failure; NIV = noninvasive ventilation.

Secondary outcome measures included dyspnea sensation, $\mathrm{P}_{\text {Imax }}$, maximum expiratory pressure $\left(\mathrm{P}_{\mathrm{Emax}}\right)$, 6-min walk distance (6MWD), BODE (body mass index, air-flow obstruction, dyspnea, exercise capacity) index, and quality of life score using the 36-Item Short Form Health Survey (SF-36; RAND Health, Santa Monica, California).

\section{Procedures}

All evaluations were performed by trained professionals in the Physiotherapy Department at the Federal University of São Carlos in São Carlos, São Paulo, Brazil. Maximal and submaximal exercise tests were performed to evaluate exercise capacity, respiratory muscle strength was measured, the BODE index was calculated, and the SF-36 quality of life survey was administered. The tests were performed by examiners who did not know the subjects' allocation to the studied groups.

Incremental Cardiopulmonary Exercise Testing. Exercise tests were performed in the afternoon to avoid different physiological responses due to circadian changes. ${ }^{14}$ Tests were performed on a treadmill (ATL, Inbrasport, Rio Grande do Sul, Brazil) according to a protocol previously applied by our group. ${ }^{23}$ After calibration according to the manufacturer's recommendations, ventilatory and metabolic measurements were obtained with a metabolic system (Oxycon Mobile, Jaeger, Hoechberg, Germany).
Breathing frequency, maximum $\dot{\mathrm{V}}_{\mathrm{O}_{2}}$, carbon dioxide output $\left(\dot{\mathrm{V}}_{\mathrm{CO}_{2}}\right)$, and minute ventilation $\left(\dot{\mathrm{V}}_{\mathrm{E}}\right)$ were recorded throughout the test. The mean of the highest values reached during the incremental test's final $30 \mathrm{~s}$ was computed. $\dot{\mathrm{V}}_{\mathrm{E}}$ and $\dot{\mathrm{V}}_{\mathrm{CO}_{2}}$ values acquired from exercise initiation to peak were entered into spreadsheet software (Microsoft, Bellevue, Washington) to calculate the slope. ${ }^{24}$ Circulatory power was defined as the product of maximum $\dot{\mathrm{V}}_{\mathrm{O}_{2}}$ and peak systolic blood pressure. Exercise ventilatory power was defined as peak systolic blood pressure divided by the $\dot{\mathrm{V}}_{\mathrm{E}} / \dot{\mathrm{V}}_{\mathrm{CO}_{2}}$ slope. ${ }^{24}$ In addition, arterial blood samples were drawn and analyzed using a lactimeter (YSI1500 Sport, YSI Life Sciences, Yellow Springs, Ohio) as previously described. ${ }^{25}$ Tests were terminated according to previously established criteria. ${ }^{14} \mathrm{~S}_{\mathrm{pO}_{2}}$, heart rate, and blood pressure were also obtained. Subjects were asked to indicate their shortness of breath using a modified Borg dyspnea scale.

Constant Speed Exercise Testing. At $48 \mathrm{~h}$ after the previous maximal exercise test, a constant speed test was applied at $80 \%$ of the maximum velocity reached in the cardiopulmonary exercise testing to assess the physiological effects of submaximal exercise during $6 \mathrm{~min}$. The same intensity was applied after the PT program. Ventilatory and metabolic measurements (ie, $\mathrm{S}_{\mathrm{pO}_{2}}$ and heart rate) were continuously monitored, and dyspnea scores (CR10 Borg scale) were obtained at the end of the exercise.

6-Minute Walk Test (6MWT). The 6MWT was applied according to the American Thoracic Society standardization, using a $30-\mathrm{m}$ corridor. ${ }^{26}$ Each subject was instructed to walk as far as possible during the allotted time, and all subjects were given standardized encouragement during the test. We conducted the 6MWT to assess the subjects' tolerance for physical effort because it is easy to apply and is usually well tolerated; moreover, the test is a good simulation of a daily activity, which makes it possible to evaluate performance of daily life activities.

Respiratory Muscle Strength Measurements. $\mathrm{P}_{\mathrm{Imax}}$ and $\mathrm{P}_{\text {Emax }}$ were assessed using a manometer (Ger-Ar Med, Sao Paulo/Brazil) as previously described. ${ }^{14}$ The predicted values were determined by the equations proposed by Neder et al. ${ }^{27}$

BODE Index. The multidimensional grading system was calculated using the following variables: body composition by body mass index, degree of airway obstruction by $\mathrm{FEV}_{1}$ with respect to the predicted value, dyspnea by the Modified Medical Research Council dyspnea scale, and ability to exercise by walking distance in the 6MWT. The subjects received scores according to the results obtained for each of the 4 variables ( $0-3$ for $\mathrm{FEV}_{1}$, dyspnea, and 


\section{Exercise Training With NIV in Subjects With COPD}

6MWD; $0-1$ for body mass index), and these results were added to obtain a total score that ranged from 0 to 10 . The higher the score, the greater the likelihood of mortality. ${ }^{28}$ Subject classification was divided into quartiles.

SF-36 Survey. Quality of life was assessed by a generic questionnaire, validated for the Portuguese language ${ }^{29}$ and covering 8 domains: functional capacity, physical aspects, pain, general health, vitality, social aspects, emotional aspect, and mental health. The total score range is $0-100$ $(0=$ worst quality of life, $100=$ best quality of life $)$. The questionnaire application is described elsewhere. ${ }^{29}$

\section{Titration of Pressure Levels and Adaptation to the Interface}

Before starting the physical training program, subjects in the NIV-Physical training group performed an NIV adaptation protocol to establish training pressure levels and to get used to the interface. NIV was delivered with a bi-level PAP ventilator (BiPAP-S, Respironics, Murrysville/Pennsylvania) via a tightly fitting interface. The adaptation period took place over 3 visits. With the subject at rest, inspiratory pressure was initially set at $6 \mathrm{~cm} \mathrm{H}_{2} \mathrm{O}$, and then it was gradually increased by increments of $2 \mathrm{~cm} \mathrm{H}_{2} \mathrm{O}$, until the subject was able to breathe naturally without additional increases in breathing frequency, signs of discomfort, or airway leaks. Expiratory pressure was set at $3 \mathrm{~cm} \mathrm{H}_{2} \mathrm{O}$, and then it was gradually increased by increments of $1 \mathrm{~cm} \mathrm{H}_{2} \mathrm{O}$, until the subject did not contract the expiratory musculature, indicated discomfort, or showed signs of air leakage. ${ }^{14}$ After a resting period $( \pm 10 \mathrm{~min}$ ), subjects were instructed to walk at the speed set for their training, with the preselected pressure levels and interface $( \pm 10 \mathrm{~min})$. If necessary, values were adjusted according to the subject's tolerance. In this study, differences between the inspiratory and expiratory pressure levels were always kept at $>5 \mathrm{~cm} \mathrm{H}_{2} \mathrm{O}$. All subjects opted for the nasal mask; 12 subjects did not tolerate the facial mask, and 17 subjects could not walk longer than 5 min due to airway dryness provoked by the interface.

\section{Intervention}

PT was applied in both groups for 6 weeks, 3 times/ weeks, for a total of 18 sessions. Each session consisted of $5 \mathrm{~min}$ of stretching for the cervical muscles and the upper and lower limbs, followed by $5 \mathrm{~min}$ of warm-up on a treadmill at $2 \mathrm{~km} / \mathrm{h}$, and then $30 \mathrm{~min}$ of physical training at $70-80 \%$ of the maximum speed reached in the cardiopulmonary exercise testing. ${ }^{23}$ The sessions were conducted individually and supervised by a team of physiotherapists. The speed was increased during the following weeks ac- cording to exercise tolerance and did not differ between groups $(P>.05$, data not shown). Oxygen supplementation was required only in those subjects who had desaturation during exercise $<90 \%$. Subjects in the NIV-Physical training group were instructed to walk with the NIV system comfortably, to breathe through the nose, and not to speak or open their mouths so that they would not allow air to escape through the mouth. Subjects were also instructed to raise their hands in case of any warning signs or symptoms during training. Subjects were required to complete at least $80 \%$ of the sessions of the proposed program to be considered for subsequent analysis.

\section{Statistical Analysis}

Considering an $\alpha$ error of 5\% and a power of $90 \%$, the minimum size required for the intervention study to be performed was determined to be 32 subjects (16 in each group). However, considering possible losses or failure to complete $80 \%$ of the program, the size was increased by $30 \%$. Thus, a minimum of 42 subjects were considered for this study, with approximately 21 subjects in each group. The researcher who performed the statistical analysis was blinded to which group subjects belonged to.

Data distribution was assessed using the Shapiro-Wilk normality test. The chi-square statistic was used to test the variance between categorical variables. A 2-way analysis of variance was applied to assess the effect of 2 factors and the interaction between them. The considered factors were time (pre-intervention vs post-intervention) and intervention (physical training vs NIV-Physical training), as well as the interaction between time and intervention. If the interaction was significant, the estimated marginal means test was applied. Because observations were performed on the same individual more than once, repeat measures tests were applied.

Within-group effect sizes (pre- to post-intervention changes) were calculated using Cohen's $d$ and interpreted as trivial (0-0.19), small (0.20-0.49), medium (0.50$0.79)$, or large $(\geq 0.80)$ effect sizes. ${ }^{30}$ Data were presented as mean $\pm \mathrm{SD}$ or as absolute and relative frequency. The level of significance was 5\%. Statistical analysis was performed using SPSS 13.0 software (SPSS, Chicago, Illinois).

\section{Results}

\section{Population Characteristics}

Of the 47 subjects included in the study, 4 were excluded: 3 dropped out of the study due to $<80 \%$ adherence to the program), and 1 presented an exacerbation during the training program (Fig. 1). Five subjects in the physical training group and 3 subjects in the NIV-Physical training 


\section{Exercise Training With NIV in Subjects With COPD}

Table 1. Subject Characteristics and Pulmonary Function Data

\begin{tabular}{|c|c|c|c|}
\hline & Physical training & NIV-Physical training & $P$ \\
\hline Sex, $n$ (female/male) & $4 / 18$ & $4 / 17$ & .99 \\
\hline Age, y & $68.2 \pm 8.5$ & $67.8 \pm 8.9$ & .99 \\
\hline Body mass, $\mathrm{kg}$ & $65.5 \pm 12.3$ & $60.5 \pm 13.4$ & .12 \\
\hline Height, cm & $163.9 \pm 8.6$ & $162.3 \pm 6.7$ & .48 \\
\hline Body mass index, $\mathrm{kg} / \mathrm{m}^{2}$ & $24.3 \pm 3.7$ & $23 \pm 5.2$ & .22 \\
\hline $\mathrm{FEV}_{1} / \mathrm{FVC}$ & $46.6 \pm 10.6$ & $46.6 \pm 11.3$ & .99 \\
\hline $\mathrm{FEV}_{1}, \mathrm{~L}$ & $1.1 \pm 0.4$ & $1 \pm 0.4$ & .37 \\
\hline $\mathrm{FEV}_{1}, \%$ pred & $41.4 \pm 14.6$ & $38.7 \pm 16.3$ & .45 \\
\hline FVC, L & $2.1 \pm 0.6$ & $2.0 \pm 0.8$ & .62 \\
\hline FVC, \% pred & $67.7 \pm 16.5$ & $65.5 \pm 22.6$ & .83 \\
\hline $\mathrm{P}_{\text {Imax }}, \mathrm{cm} \mathrm{H}_{2} \mathrm{O}(\%$ pred $)$ & $46 \pm 24(46 \pm 21)$ & $44 \pm 25(45 \pm 25)$ & .82 \\
\hline $\mathrm{P}_{\text {Emax }}, \mathrm{cm} \mathrm{H}_{2} \mathrm{O}(\%$ pred $)$ & $72 \pm 28(72 \pm 25)$ & $67 \pm 22(64 \pm 19)$ & .78 \\
\hline Domiciliary oxygen therapy, $n$ & $5(20.8)$ & $3(13)$ & NA \\
\hline \multicolumn{4}{|c|}{$\begin{array}{l}\text { Data are presented as } n \text { subjects or mean } \pm \text { SD. There were no significant differences between groups. } \\
\text { For the physical training group, } n=22 \text { subjects; for the NIV-Physical training group, } n=21 \text { subjects. } \\
\text { NIV = noninvasive ventilation } \\
\mathrm{P}_{\text {Imax }}=\text { maximum inspiratory pressure } \\
\mathrm{P}_{\text {Emax }}=\text { maximum expiratory pressure } \\
\text { NA = not applicable }\end{array}$} \\
\hline
\end{tabular}

grouped used domiciliary oxygen therapy at night. None of the subjects used domiciliary oxygen therapy for 12$24 \mathrm{~h}$. Of the physical training group, 22 subjects completed the physical training program, and 21 subjects completed the NIV-Physical training program.

All of the subjects had moderate to very severe airflow obstruction per GOLD. In the physical training group, 4 subjects were stage II, 12 subjects were stage III, and 6 subjects were stage IV GOLD stage. In the NIV-Physical training groups, 4 subjects were stage II, 11 subjects were stage III, and 7 subjects were stage IV. All subjects presented with reduced maximal exercise capacity (ie, maximum $\mathrm{V}_{\mathrm{O}_{2}}$ below the lower limit of normality). Breathlessness was an exercise-limiting symptom in all subjects. All subjects presented inspiratory muscle weakness $\left(\mathrm{P}_{\text {Imax }}<70 \%\right.$ of predicted) (Table 1$)$.

In the physical training group, 5 subjects received supplemental oxygen via dual-prong nasal cannula at $1-2 \mathrm{~L} / \mathrm{min}$, which was determined to be the minimum flow sufficient to keep fingertip saturation $>90 \%$. Only 3 subjects of the NIVPhysical training group required supplemental oxygen, and this was delivered through the nasal mask at $0.5-1 \mathrm{~L} / \mathrm{min}$ $(P<0.05$, data not shown).

All subjects successfully tolerated NIV during exercise, and no subjects discontinued due to NIV. Mean \pm SD values for inspiratory and expiratory pressures during the physical training program were $12 \pm 2 \mathrm{~cm} \mathrm{H}_{2} \mathrm{O}$ and $5 \pm 1 \mathrm{~cm} \mathrm{H}_{2} \mathrm{O}$, respectively.

The physical training program had significant positive effects on maximum performance in both groups (time effect), as demonstrated by test time $(P<.001)$, 6MWD $(P<.001)$, maximum speed $(P<.001)$, maximum $\dot{\mathrm{VCO}}_{2}$
$(P=.005)$, and exercise ventilatory power $(P=.04)$ (Table 2). However, maximum $\dot{\mathrm{VO}}_{2}$ (Fig. 2) and peak metabolic equivalents significantly increased only in the NIV-Physical training group (time and interaction effect, $P=.01$ for both). Consequently, circulatory power significantly increased only in the NIV-Physical training group (interaction effect, $P=.006$ ).

Table 2 shows that the peak dyspnea in the NIV-Physical training group was associated with lower values after exercise (time and interaction effects, $P=.04$ ). Blood lactate significantly decreased after physical training only in the NIV-Physical training group (time, group, and interaction effects, $P=.004$ ). In addition, peak $\mathrm{SpO}_{2}$ was significantly reduced after the training program only for the physical training group (time and interaction effects, $P=.02$ ).

With regard to the constant speed testing (Table 3$), \dot{\mathrm{V}}_{\mathrm{E}}$ $(P=.03)$, dyspnea $(P<.001)$ and heart rate $(P=.02)$ significantly decreased in both groups (time effect). However, the groups differed after the training program, with significantly higher $\mathrm{SpO}_{2}$ values only for the NIV-Physical training group (group effect, $P=.04$ ).

Table 4 shows the results of the PT program for the 2 groups. The program effects were not different between the groups, with increases in PImax $(P<.001)$ and 6MWD $(P<.001)$, as well as a reduced BODE index $(P=.03)$. In addition, the exercise program resulted in an improvement of quality of life in the social $(P<.001)$ and emotional $(P=$ .04) domains, with no differences between groups. Interestingly, however, PEmax significantly increased only for the NIV-Physical training group $(P=.002)$, with an interaction between time and group $(P=.004)$. 


\section{Exercise Training With NIV in Subjects With COPD}

Table 2. Effects of Physical Training and of NIV + Physical Training on Maximal Performance During Exercise

\begin{tabular}{|c|c|c|c|c|c|c|c|}
\hline & \multicolumn{2}{|c|}{ Physical training } & \multicolumn{2}{|c|}{ NIV-Physical training } & \multicolumn{3}{|c|}{$P$} \\
\hline & Pre & Post & Pre & Post & Time & Group & Interaction \\
\hline Maximum time, min & $10 \pm 4.5$ & $12.4 \pm 5.1 *$ & $9.2 \pm 5.8$ & $12.4 \pm 5.9 *$ & $<.001$ & .84 & .31 \\
\hline 6-min walk distance, $\mathrm{m}$ & $435.3 \pm 200$ & $613.5 \pm 273.4^{*}$ & $440.2 \pm 363.5$ & $710 \pm 440.1^{*}$ & $<.001$ & .66 & .15 \\
\hline Maximum speed, km/h & $3.5 \pm 0.8$ & $4.2 \pm 0.9^{*}$ & $3.5 \pm 1.2$ & $4.4 \pm 1.4^{*}$ & $<.001$ & .82 & .27 \\
\hline Maximum $\dot{V}_{\mathrm{E}}, \mathrm{L} / \mathrm{min}$ & $25.7 \pm 7.1$ & $25.9 \pm 9.3$ & $20.6 \pm 4.3$ & $25.5 \pm 7.3$ & .07 & .18 & .09 \\
\hline Maximum $\dot{\mathrm{V}}_{\mathrm{O}_{2}}, \mathrm{~mL} / \mathrm{kg} / \mathrm{min}$ & $14.3 \pm 4.3$ & $14.8 \pm 3.4$ & $13.2 \pm 4$ & $18.1 \pm 5.7 * \div$ & .01 & .41 & .003 \\
\hline Maximum $\dot{\mathrm{V}}_{\mathrm{CO}_{2}}, \mathrm{~L} / \mathrm{min}$ & $1 \pm 0.3$ & $1.1 \pm 0.4^{*}$ & $0.8 \pm 0.3$ & $1.1 \pm 0.5^{*}$ & .005 & .57 & .09 \\
\hline Maximum $\dot{\mathrm{V}}_{\mathrm{O}_{2}} / \dot{\mathrm{V}}_{\mathrm{CO}_{2}}$ & $1.1 \pm 0.1$ & $1 \pm 0.2$ & $1 \pm 0.1$ & $1 \pm 0.2$ & .24 & .89 & .29 \\
\hline Maximum metabolic equivalents & $3.8 \pm 1.3$ & $3.9 \pm 1.1$ & $3.6 \pm 1.3$ & $5 \pm 1.6^{*}+$ & .01 & .25 & .02 \\
\hline$\dot{\mathrm{V}}_{\mathrm{E}} / \dot{\mathrm{V}}_{\mathrm{CO}_{2}}$ slope & $28.4 \pm 4.9$ & $27 \pm 5.1$ & $28.3 \pm 5.3$ & $25.5 \pm 6.4$ & .09 & .59 & .55 \\
\hline Dyspnea $(0-10)$ & $5.1 \pm 0.25$ & $5.5 \pm 1.4$ & $5.8 \pm 0.87$ & $4.2 \pm 1.7 * \div$ & .04 & .63 & .001 \\
\hline Maximum heart rate, beats/min & $112.8 \pm 11.7$ & $115.9 \pm 14.6$ & $111.8 \pm 14.2$ & $118.2 \pm 17.1$ & .06 & .89 & .49 \\
\hline Maximum $\mathrm{S}_{\mathrm{pO}_{2}}, \%$ & $86.3 \pm 5.1$ & $83.4 \pm 6.3$ & $85.5 \pm 5.8$ & $86.1 \pm 4.5 \ddagger$ & .12 & .61 & .02 \\
\hline Blood lactate, $\mu \mathrm{mol} / \mathrm{L}$ & $2.49 \pm 1.0$ & $2.63 \pm 0.92$ & $2.63 \pm 0.75$ & $1.90 \pm 0.87 *+\neq$ & .004 & .040 & .01 \\
\hline $\mathrm{EVP}, \mathrm{mm} \mathrm{Hg}$ & $4.6 \pm 1$ & $5.7 \pm 2.6^{*}$ & $4.2 \pm 1.3$ & $4.7 \pm 1.7 *$ & .04 & .17 & .42 \\
\hline $\mathrm{CP}, \mathrm{mm} \mathrm{mmHg} / \mathrm{mL} \mathrm{O}_{2} / \mathrm{kg} / \mathrm{min}$ & $2644 \pm 810$ & $2601 \pm 737 *$ & $2165 \pm 729$ & $3059 \pm 243 * \ddagger$ & .006 & .97 & .003 \\
\hline $\begin{array}{l}\text { Values are presented as mean } \pm \mathrm{SD} \text {. } \\
\text { For the physical training group, } n=22 \text { subj } \\
\mathrm{NIV}=\text { noninvasive ventilation } \\
\mathrm{V}_{\mathrm{E}}=\text { minute ventilation } \\
\dot{\mathrm{V}}_{\mathrm{O}_{2}}=\text { oxygen consumption } \\
\dot{\mathrm{V}}_{\mathrm{CO}_{2}}=\text { carbon dioxide production } \\
\mathrm{EVP}=\text { maximum systolic blood pressure di } \\
\mathrm{CP}=\text { circulatory power (product of maximu } \\
* \text { Significant difference for time effect }(P< \\
\dagger \text { Significant difference between groups effe } \\
\text { † Significant interaction between group and }\end{array}$ & $\begin{array}{l}\mathrm{d} \text { by the } \mathrm{V}_{\mathrm{E}} / \dot{\mathrm{V}}_{\mathrm{CO}_{2}} \mathrm{sl} \\
\mathrm{O}_{2} \text { and maximum sy } \\
\text { fter training }(P<.05 \\
\text { effects }(P<.05) \text {. }\end{array}$ & lic blood pressure) & & & & & \\
\hline
\end{tabular}

\section{Discussion}

This study shows that a physical training program positively affected exercise tolerance, some aspects of quality of life, and BODE index scores in subjects with moderate to very severe COPD; however, NIV in conjunction with a physical training program demonstrates additional beneficial effects on maximum $\dot{\mathrm{V}}_{\mathrm{O}_{2}}$, blood lactate, dyspnea, and peak circulatory power during maximal exercise, on $\mathrm{S}_{\mathrm{pO}_{2}}$ during submaximal exercise, and on $\mathrm{P}_{\text {Emax }}$. From a clinical perspective, our data indicate that strategies aimed at reducing work of breathing, such as NIV, can have additional beneficial effects on important physiological markers and symptoms during exercise in this population.

\section{Methodological Considerations}

In this study, we chose to apply NIV instead of other modes because it has been used frequently in previous studies. ${ }^{15-20}$ Our study strengthens the evidence on the effects of NIV as an adjunct in rehabilitating patients with COPD. The benefits on exercise capacity provided by NIV have been recognized for patients with COPD; $;^{14,20,31}$ in addition, even though no studies have evaluated costs, NIV is the most economical modality, compared to pressure support ventilation or proportional assist ventilation. Effective options that involve lower costs should be encouraged in future studies.

As for the interface, we let each subject choose what was most comfortable for them. We offered facial (oronasal), nasal, and oral masks; $100 \%$ of subjects opted for the nasal mask. In the study by Van't Hul et al, ${ }^{19}$ the authors applied NIV by mouthpiece and did not observe any related discomfort. Other authors have argued that nasal masks could influence results because of the subjects' need to open their mouth to breathe when high effort is demanded. ${ }^{31}$ In our study, the training team reported that no subject needed to open their mouth to breathe during exercise.

Another important aspect to be considered was the type of exercise to be performed by subjects with COPD. Some authors have applied exercise on a cycle ergometer, ${ }^{15,19}$ while others have applied exercise on a treadmill. ${ }^{14,20}$ Previous studies have shown that exercise on a treadmill exerts a greater ventilatory and metabolic demand and induces more desaturation than exercise performed on a cycle ergometer. ${ }^{32,33}$ Another study has 


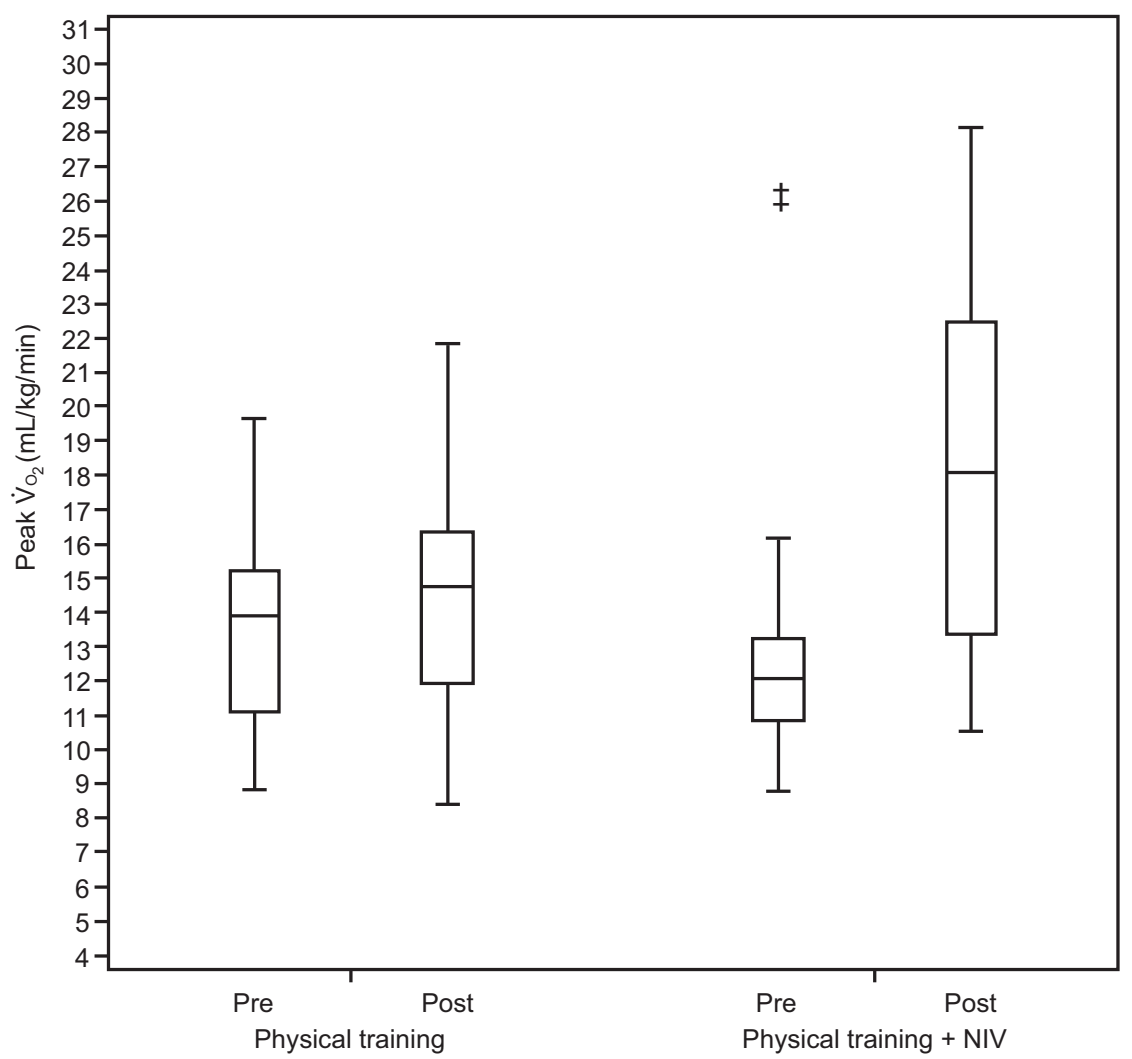

Fig. 2. Differences in maximum oxygen consumption $\left(\dot{\mathrm{V}}_{\mathrm{O}_{2}}\right)$ during physical training and noninvasive ventilation (NIV) + Physical training, during maximum cardiopulmonary exercise testing on a treadmill. $\ddagger$ Significant interaction between group and time effects $(P<.05)$.

Table 3. Effects of Physical Training and of NIV + Physical Training on Submaximal Performance

\begin{tabular}{|c|c|c|c|c|c|c|c|}
\hline & \multicolumn{2}{|c|}{ Physical training } & \multicolumn{2}{|c|}{ NIV-Physical training } & \multicolumn{3}{|c|}{$P$} \\
\hline & Pre & Post & Pre & Post & Time & Group & Interaction \\
\hline$\dot{\mathrm{V}}_{\mathrm{E}}, \mathrm{L} / \mathrm{min}$ & $25 \pm 7.7$ & $19.4 \pm 6.6^{*}$ & $24.8 \pm 10.1$ & $20.3 \pm 7 *$ & .030 & .90 & .81 \\
\hline$\dot{\mathrm{V}}_{\mathrm{O}_{2}}, \mathrm{~L} / \mathrm{min}$ & $1 \pm 0.3$ & $0.8 \pm 0.3$ & $0.7 \pm 0.2$ & $0.7 \pm 0.3$ & .07 & .30 & .49 \\
\hline$\dot{\mathrm{V}}_{\mathrm{CO}_{2}}, \mathrm{~L} / \mathrm{min}$ & $1.1 \pm 0.5$ & $0.7 \pm 0.4$ & $0.6 \pm 0.2$ & $0.7 \pm 0.2$ & .18 & .12 & .13 \\
\hline Metabolic equivalents & $4.2 \pm 1.3$ & $3.5 \pm 1.7$ & $3.3 \pm 1.1$ & $3.6 \pm 1.7$ & .69 & .59 & .18 \\
\hline Dyspnea & $2.7 \pm 1.6$ & $1.1 \pm 1.6^{*}$ & $4.5 \pm 2.1$ & $1.4 \pm 2 *$ & $<.001$ & .09 & .13 \\
\hline Heart rate, beats/min & $106.6 \pm 15.6$ & $104.4 \pm 13.1 *$ & $112.2 \pm 20.5$ & $103.2 \pm 19.5^{*}$ & .02 & .75 & .14 \\
\hline $\mathrm{S}_{\mathrm{pO}_{2}}$ at rest, $\%$ & $92.3 \pm 3.8$ & $93.2 \pm 1.7$ & $94.3 \pm 3.4$ & $95.5 \pm 2.1$ & .26 & .060 & .89 \\
\hline $\mathrm{S}_{\mathrm{pO}_{2}}, \%$ & $84.5 \pm 5.5$ & $86.3 \pm 5.2$ & $89.8 \pm 4.5$ & $90 \pm 4.6 \dagger$ & .37 & .040 & .45 \\
\hline $\begin{array}{l}\text { Values are presented as mean } \\
\text { For the physical training grour } \\
\text { NIV = noninvasive ventilation } \\
\dot{V}_{\mathrm{E}}=\text { minute ventilation } \\
\mathrm{V}_{\mathrm{O}_{2}}=\text { oxygen consumption } \\
\mathrm{V}_{\mathrm{CO}_{2}}=\text { carbon dioxide produ } \\
\text { * Significant difference for tin } \\
\dagger \text { Significant difference betwe } \\
\text { Due to this test performed not }\end{array}$ & $\begin{array}{l}\text { et }(\mathrm{p}<.05) \\
\text { ps effect after train } \\
\text { significant interact }\end{array}$ & ysical training group & , the post test the & was used. & & & \\
\hline
\end{tabular}

shown that cycle ergometer exercise, which was unfamiliar to the participating subjects, generated a high lactate response. ${ }^{34}$ In our study, we chose to apply exercise on a treadmill because, in addition to potentiating performance gains by involving more muscle mass, walking is a type of exercise that recruits larger muscle groups and, therefore, imposes a greater metabolic and ventilatory demand. 
Table 4. Effects of Physical Training and of NIV + Physical Training

\begin{tabular}{|c|c|c|c|c|c|c|c|}
\hline & \multicolumn{2}{|c|}{ Physical training } & \multicolumn{2}{|c|}{ NIV-Physical training } & \multicolumn{3}{|c|}{$P$} \\
\hline & Pre & Post & Pre & Post & Time & Group & Interaction \\
\hline $\mathrm{P}_{\text {Imax }}, \mathrm{cm} \mathrm{H}_{2} \mathrm{O}$ & $45.9 \pm 24.2$ & $57.5 \pm 24.7^{*}$ & $44.2 \pm 25.7$ & $65 \pm 30.9 *$ & $<.001$ & .70 & .13 \\
\hline $\mathrm{P}_{\mathrm{Emax}}, \mathrm{cm} \mathrm{H}_{2} \mathrm{O}$ & $74.3 \pm 29.2$ & $75 \pm 28.8$ & $67.1 \pm 23$ & $86.1 \pm 32.9 * \dagger$ & .002 & .80 & .004 \\
\hline 6-min walk distance, $\mathrm{m}$ & $374.8 \pm 115.2$ & $419.2 \pm 108.1^{*}$ & $363 \pm 158$ & $451.5 \pm 158.2^{*}$ & $<.001$ & .79 & .12 \\
\hline BODE index, total score & $1.95 \pm 0.7$ & $1.86 \pm 0.6^{*}$ & $2.32 \pm 0.8$ & $2.04 \pm 0.7 *$ & .030 & .27 & .17 \\
\hline \multicolumn{8}{|l|}{ Short Form-36 } \\
\hline Physical functioning & $63.3 \pm 24.5$ & $70.7 \pm 17.3^{*}$ & $58.1 \pm 27.8$ & $70.8 \pm 27.1^{*}$ & .004 & .74 & .42 \\
\hline Role limitations as a result of physical problems & $70.8 \pm 39.5$ & $70.8 \pm 40.4$ & $58.1 \pm 48.5$ & $76.9 \pm 37.4$ & .09 & .50 & .09 \\
\hline Body pain & $68.8 \pm 24.9$ & $78 \pm 21.2$ & $79.2 \pm 27.8$ & $76.8 \pm 30$ & .52 & .53 & .28 \\
\hline General health perception & $65.1 \pm 20$ & $69.5 \pm 17.1$ & $60.2 \pm 26$ & $56.7 \pm 19.8$ & .90 & .18 & .24 \\
\hline Vitality & $59.8 \pm 20.3$ & $62.6 \pm 16.4$ & $58.1 \pm 22.8$ & $56.9 \pm 24.6$ & .75 & .59 & .46 \\
\hline Social functioning & $76.8 \pm 27.8$ & $89.3 \pm 17.8^{*}$ & $71.9 \pm 24.5$ & $94.8 \pm 14.6^{*}$ & $<.001$ & .97 & .26 \\
\hline Role limitations as a result of emotional problems & $83.3 \pm 28.6$ & $87 \pm 23.3^{*}$ & $81.8 \pm 31.1$ & $100 \pm 0.0^{*}$ & .04 & .47 & .18 \\
\hline Mental health & $67.2 \pm 22.3$ & $72.4 \pm 19.9$ & $64.6 \pm 20.6$ & $67.1 \pm 17.2$ & .19 & .55 & .64 \\
\hline $\begin{array}{l}\text { Values are presented as mean } \pm \mathrm{SD} \text {. } \\
\text { For the physical training group, } n=22 \text { subjects; for the NIV-Physical } \\
\mathrm{NIV}=\text { noninvasive ventilation } \\
\mathrm{P}_{\text {Imax }}=\text { maximum inspiratory pressure } \\
\mathrm{P}_{\text {Emax }}=\text { maximum expiratory pressure } \\
* \text { Significant difference for time effect }(P<.05) \text {. } \\
\dagger \text { Significant interaction between group and time effects }(P<.05) \text {. } \\
\text { For the variables that presented interaction significant was used the pos }\end{array}$ & training group, $n=21$ & subjects. & ( & 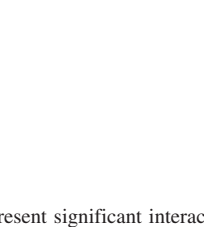 & 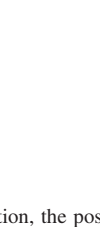 & 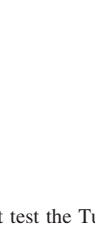 & \\
\hline
\end{tabular}

\section{Effects of a 6-week Physical Training Program for Subjects With COPD}

The proposed 6-week physical training program on a treadmill proved to be beneficial to subjects with moderate to very severe COPD, with a positive effect on exercise tolerance, $\mathrm{P}_{\text {Imax }}$, and quality of life. When considering maximal and submaximal exercise, an improvement was observed for some ventilatory variables in both tests, in addition to a heart rate reduction in the constant speed test.

According to the vast literature on the effects of exercise, an initial adaptation occurs in the skeletal muscles, ${ }^{35}$ which could allow subjects with COPD to exercise with reduced ventilatory demand while maintaining the same work load. ${ }^{9}$ Our results, which include a significant reduction in the ventilatory demand during submaximal exercise intensities, corroborate those data.

In addition, the 6-week physical training program also led to an increased $\mathrm{P}_{\text {Imax }}$ in both groups. Subjects enrolled in this study presented inspiratory muscle weakness $(<70 \%$ of predicted values), which could help explain their marked exercise intolerance. ${ }^{36}$ Another benefit of the physical training program in our study was better ventilatory efficiency as indicated by an improvement in exercise ventilatory power, which is an important marker of morbidity and mortality in congestive heart failure. ${ }^{24}$ These results are relevant because this index has been poorly explored after the application of a physical training program in subjects with COPD.
Another important consideration to note is the intervention time proposed in our study. Short-term physical training programs, usually 6 or 8 weeks long, were able to interrupt the vicious cycle of dyspnea and intolerance to physical exertion. ${ }^{7}$ According to the data discussed in the meta-analysis conducted by McCarthy et al, ${ }^{37}$ an average increase in 6MWD of $43 \mathrm{~m}$ after a pulmonary rehabilitation program is considered a clinically important difference and indicates that exercise improved the subjects' functional capacity.

It is worth mentioning that exercise is considered one of the pillars of pulmonary rehabilitation of patients with COPD because it determines an improvement in functional capacity, reflected by increases in 6MWD and quality of life..$^{3,37}$ Regarding the quality of life of the subjects enrolled in our study, results showed that improved exercise tolerance after the physical training program had positive effects on the quality of life of subjects in both groups in the functional, social, and emotional domains, similar to previous studies.

Márquez-Martín et al $^{38}$ observed an improvement in the BODE index and the quality of life in subjects with COPD when performing exercise alone as well as when exercise was combined with nighttime NIV for 12 weeks, with no other differences between the protocols. In our study, these variables presented an improvement with both protocols after only 6 weeks, with no differences between groups. 


\section{Exercise Training With NIV in Subjects With COPD}

\section{Additional Effects of Bi-Level NIV After a 6-week Physical Training Program in Subjects With Moderate to Very Severe COPD}

In this study, we observed significant maximum $\dot{\mathrm{V}}_{\mathrm{O}_{2}}$ and peak metabolic equivalent improvements only in the NIV-Physical training group. $\dot{\mathrm{O}}_{\mathrm{O}_{2}}$ is an important marker of true physiological adaptation to exercise in patients with COPD. ${ }^{39}$ The gain in exercise capacity indicated by the maximum $\dot{\mathrm{V}}_{\mathrm{O}_{2}}$ increase of $4.9 \mathrm{~mL} / \mathrm{kg} / \mathrm{min}$ observed in this study is probably due to the use of NIV because the use of positive pressure tends to improve cardiac function and regional vascular distribution, in addition to reducing the transmural pressure gradient and the left ventricular afterload, which allows the improvement of blood supply to the periphery, subsequently allowing individuals to gain exercise capacity. ${ }^{40}$

Carvalho et $\mathrm{al}^{40}$ showed that the minimum detectable difference calculated for maximum $\dot{\mathrm{V}}_{\mathrm{O}_{2}}$ was $1.02 \mathrm{~mL} / \mathrm{kg} / \mathrm{min}$. A maximum $\dot{\mathrm{V}}_{\mathrm{O}_{2}}$ increase of $2.2 \mathrm{~mL} / \mathrm{kg} / \mathrm{min}$ was observed, corresponding to a gain of $12.3 \%$ in the NIV phase compared with the control phase.

In a previous study by our group, we showed that exercise alone significantly improved maximum $\dot{\mathrm{V}}_{\mathrm{O}_{2}}$ after only 12 weeks. ${ }^{41}$ Another study comparing NIV and oxygen supplementation showed that only NIV could improve maximum $\dot{\mathrm{V}}_{\mathrm{O}_{2}}$ after 6 weeks. ${ }^{14}$ These results, taken together, confirm that NIV can help sustain higher levels of training intensity, which can allow a short-term physical training program to have positive effects on the enzymatic, mitochondrial, and vascular mechanics of peripheral musculature. ${ }^{17}$ However, muscular intrinsic factors were not explored in the present study. We also observed a significant reduction in blood lactate, another important factor that limits exercise capacity, especially in subjects with more severe COPD. ${ }^{14,20,42}$

We observed a significant improvement in circulatory power only in the NIV-Physical training group. Circulatory power represents the volume of $\mathrm{O}_{2}$ added to the mixed venous blood by the lungs and transferred to systemic arterial circulation, against a pressure gradient, by the heart. ${ }^{43}$ In this context, circulatory power has been proposed as a surrogate of cardiac power because it presented a better prognostic value than maximum $\dot{\mathrm{V}}_{\mathrm{O}_{2}}, \mathrm{O}_{2}$ pulse, or peak systolic blood pressure in subjects with chronic heart disease. ${ }^{43}$ However, to our knowledge, no studies have focused on the effects of a physical training program in combination with NIV on circulatory power. Considering the current literature on other chronic diseases, circulatory power appears to be a promising parameter to evaluate the effects of nonpharmacological interventions in patients with COPD.

As previously discussed, the proposal to add NIV to exercise is intended to reduce ventilatory load during high- intensity exercise, which will allow patients to exercise more vigorously, reduce ventilatory work and dyspnea symptoms, attenuate desaturation, ${ }^{14}$ and increase effort tolerance of subjects with more severe COPD. ${ }^{12,14}$

Other important outcomes that confirmed the potential beneficial effects of NIV as an adjunct to an exercise program were maximum dyspnea and peak $\mathrm{S}_{\mathrm{pO}_{2}}$. Although self-reported dyspnea is a subjective measure and $\mathrm{S}_{\mathrm{pO}_{2}}$ may be difficult to capture, especially in the presence of accelerated walking or reduced peripheral perfusion, we consider both measures to be important because of their significance and ease to obtain in clinical practice. In this study, maximum dyspnea decreased during maximal and submaximal exercise, and $\mathrm{S}_{\mathrm{pO}_{2}}$ increased only in the NIVPhysical training group. These results could be explained by considering that NIV may improve pulmonary ventilation, the ventilation-perfusion ratio, ${ }^{14}$ and dynamic hyperinflation; however, these mechanisms were not explored in depth.

In the study by Márquez-Martín et al, ${ }^{38}$ the authors compared 3 treatment protocols: NIV only, endurance training, and strength training. The combination of training with NIV for 12 weeks resulted in an increased tolerance of effort, as shown by increases in the 6MWD (eg, the authors observed a gain of $42 \mathrm{~m}$ with the isolated training, while the gain of $83 \mathrm{~m}$ was noted when the training was combined with the NIV). Similar results were observed in our study because exercise alone generated a gain of $45 \mathrm{~m}$ in the $6 \mathrm{MWD}$, while the combination with NIV led to an increase of $88 \mathrm{~m}$. This is likely due to the muscular assistance provided by the ventilatory support, which led to an increase in functional capacity.

We observed that when NIV was administered in conjunction with a PT program, expiratory muscle strength improved. We consider this result to be very important because expiratory muscle weakness has been shown to be associated with ineffective coughing and with impaired strength and work of the diaphragm. The expiratory muscle improvement we observed in this group could improve neuroventilatory dissociation during exercise. ${ }^{44}$

In the study on nocturnal NIV by Kneidinger et al,45 which involved subjects with terminal lung disease awaiting long-term pulmonary transplantation, the authors observed that pulmonary rehabilitation was feasible and provided clinically relevant improvements of physical capacity and quality of life; moreover, pulmonary rehabilitation presented increased benefits in subjects treated with NIV when compared to subjects who did not receive NIV.

The main limitation of our study was the inability to blind subjects and the physiotherapists who applied the PT program. However, we consider this limitation to be inherent to studies in which NIV and training are applied, as reported in previous studies. ${ }^{13-16,19}$ 


\section{Exercise Training With NIV in Subjects With COPD}

Another limitation of our study is that the SF-36 survey used to assess subjects' quality of life is not specific for COPD patients. However, according to the literature, this survey allows the evaluation of subjective aspects related to the disease, despite being a generic questionnaire.

A potential limitation of this study was the lack of accurate data on the final costs of physical training sessions combined with NIV. Although the low cost for NIV equipment acquisition may not be a limiting factor, the cost related to the service provided by trained professionals able to apply NIV during exercise should be considered. For these reasons, further studies are needed to investigate the possibility of successfully combining exercise programs with the application of NIV.

\section{Conclusion}

Our study has demonstrated that a 6-week exercise program alone can improve tolerance to exercise and quality of life. However, NIV in combination with an exercise program has an additive beneficial effect on powerful prognostic markers (eg, maximum $\dot{\mathrm{V}}_{\mathrm{O}_{2}}$ and circulatory power) and was able to improve oxygen saturation and reduce symptoms in subjects with moderate to very severe COPD.

\section{REFERENCES}

1. Scano G, Grazzini M, Stendardi L, Gigliotti F. Respiratory muscle energetics during exercise in healthy subjects and patients with COPD. Respir Med 2006;100(11):1896-1906.

2. Pepin V, Saey D, Laviolette L, Maltais F. Exercise capacity in chronic obstructive pulmonary disease: mechanisms of limitation. COPD 2007;4(3):195-204.

3. Vestbo J, Hurd SS, Agustí AG, Jones PW, Vogelmeier C, Anzueto A, et al. Global strategy for the diagnosis, management, and prevention of chronic obstructive pulmonary disease: GOLD executive summary. Am J Respir Crit Care Med 2013;187(4):347-365.

4. O'Donnell DE, D'Arsigny C, Fitzpatrick M, Webb KA. Exercise hypercapnia in advanced chronic obstructive pulmonary disease: the role of lung hyperinflation. Am J Respir Crit Care Med 2002;166(5): 663-668.

5. Khan NA, Daga MK, Ahmad I, Mawari G, Kumar S, Kumar N, Husain SA. Evaluation of BODE index and its relationship with systemic inflammation mediated by proinflammatory biomarkers in patients with COPD. J Inflamm Res 2016;18(9):187-198.

6. Nici L, Donner C, Wouters E, Zuwallack R, Ambrosino N, Bourbeau $\mathrm{J}$, et al. American Thoracic Society/European Respiratory Society statement on pulmonary rehabilitation. Am J Respir Crit Care Med 2006;173(12):1390-1413.

7. Casaburi R, Porszasz J, Burns MR, Carithers ER, Chang RS, Cooper CB. Physiologic benefits of exercise training in rehabilitation of patients with severe COPD. Am J Respir Crit Care Med 1997;155(5): $1541-1551$

8. Ricci C, Terzoni S, Gaeta M, Sorgente A, Destrebecq A, Gigliotti F. Physical training and noninvasive ventilation in COPD patients: a meta-analysis. Respir Care 2014;59(5):709-717.

9. Casaburi R, Patessio A, Ioli F, Zanaboni S, Donner CF, Wasserman $\mathrm{K}$. Reductions in exercise lactic acidosis and ventilation as a result of exercise training in patients with obstructive lung disease. Am Rev Respir Dis 1991;143(1):9-18.
10. Soguel Schenkel N, Burdet L, De Muralt B, Fitting JW. Oxygen saturation during daily activities in chronic obstructive pulmonary disease. Eur Respir J 1996;9(12):2584-2589.

11. O’Donnell DE, Revill SM, Webb KA. Dynamic hyperinflation and exercise intolerance in chronic obstructive pulmonary disease. Am J Respir Crit Care Med 2001;164(5):770-777.

12. Borghi-Silva A, Oliveira CC, Carrascosa C, Maia J, Berton DC, Queiroga F Jr., et al. Respiratory muscle unloading improves leg muscle oxygenation during exercise in patients with COPD. Thorax 2008;63(10):910-915.

13. Borghi-Silva A, Di Thommazo L, Pantoni CB, Mendes RG, Salvini Tde F, Costa D. Noninvasive ventilation improves peripheral oxygen saturation and reduces fatigability of quadriceps in patients with COPD. Respirology 2009;14(4):537-544.

14. Borghi-Silva A, Mendes RG, Toledo AC, Malosá Sampaio LM, da Silva TP, Kunikushita LN, et al. Adjuncts to physical training of patients with severe COPD: oxygen or noninvasive ventilation? Respir Care 2010;55(7):885-894.

15. Hawkins P, Johnson LC, Nikoletou D, Hamnegård CH, Sherwood R, Polkey MI, Moxham J. Proportional assist ventilation as an aid to exercise training in severe chronic obstructive pulmonary disease. Thorax 2002;57(10):853-859.

16. Johnson JE, Gavin DJ, Adams-Dramiga S. Effects of training with heliox and noninvasive positive pressure ventilation on exercise ability in patients with severe COPD. Chest 2002;122(2):464-472.

17. Costes F, Agresti A, Court-Fortune I, Roche F, Vergnon JM, Barthélémy JC. Noninvasive ventilation during exercise training improves exercise tolerance in patients with chronic obstructive pulmonary disease. J Cardiopulm Rehabil 2003;23(4):307-313.

18. Reuveny R, Ben-Dov I, Gaides M, Reichert N. Ventilatory support during training improves training benefit in severe chronic airway obstruction. Isr Med Assoc J 2005;7(3):151-155.

19. Van't Hul A, Gosselink R, Hollander P, Postmus P, Kwakkel G. Training with inspiratory pressure support in patients with severe COPD. Eur Respir J 2006;27(1):65-72.

20. Toledo A, Borghi-Silva A, Sampaio LM, Ribeiro KP, Baldissera V, Costa $\mathrm{D}$. The impact of noninvasive ventilation during the physical training in patients with moderate-to-severe chronic obstructive pulmonary disease (COPD). Clinics 2007;62:113-120.

21. Camillo CA, Osadnik CR, Van Remoortel H, Burtin C, Janssens W, Troosters T. Effect of "add-on" interventions on exercise training in individuals with COPD: a systematic review. ERJ Open Res 2016; 2(1):00078-2015.

22. Global Initiative for Chronic Obstructive Lung Disease (GOLD). Pocket Guide to COPD Diagnosis, Management, and Prevention. Updated January 2015.

23. Marrara KT, Marmorato D, Held PA, de Oliveira Junior AD, Jamami M, Pires Di Lorenzo VA. Different physical therapy interventions on daily physical activities in chronic obstructive pulmonary disease. Respir Med 2008;102(4):505-511.

24. Borghi-Silva A, Labate V, Arena R, Bandera F, Generati G, Pellegrino $\mathrm{M}$, et al. Exercise ventilatory power in heart failure patients: functional phenotypes definition by combining cardiopulmonary exercise testing with stress echocardiography. Int J Cardiol 2014;176(3): 1348-1349.

25. Borghi-Silva A, Baldissera V, Sampaio LM, Pires Di Lorenzo VA, Jamami M, Demonte A, et al. L-carnitine as an ergogenic aid for patients with chronic obstructive pulmonary disease submitted to whole-body and respiratory muscle training programs. Braz J Med Biol Res 2006;39(4):465-474.

26. American Thoracic Society. ATS statement: guidelines for the sixminute walk test. Am J Respir Crit Care Med 2002;166(1):111-117. 


\section{Exercise Training With NIV in Subjects With COPD}

27. Neder JA, Andreoni S, Lerario MC, Nery LE. Reference values for lung function tests. II. Maximal respiratory pressures and voluntary ventilation. Braz J Med Biol Res 1999;32(6):719-727.

28. Celli BR, Cote CG, Marín JM, Casanova C, Oca MM, et al. The body-mass index, airflow obstruction, dyspnea and exercise capacity index in chronic obstructive pulmonary disease. N Engl J Med 2004; 350:1005-1012.

29. Ciconelli RM, Ferraz MB, Santos W, Meinão I, Quaresma MR. Tradução para a língua portuguesa e validação do questionário genérico de avaliação de qualidade de vida SF-36 (Brasil SF-36). Rev Bras Reumatol 1999;39(3):143-150.

30. Fritz CO, Morris PE, Richler JJ. Effect size estimates: current use, calculations, and interpretation. J Exp Psychol Gen 2012;141(1):2-18.

31. Bianchi L, Foglio K, Porta R, Baiardi P, Vitacca M, Ambrosino N. Lack of additional effect of adjunct of assisted ventilation to pulmonary rehabilitation in mild COPD patients. Respir Med 2002;96(5): 359-367.

32. Hsia D, Casaburi R, Pradhan A, Torres E, Porszasz J. Physiological responses to linear treadmill and cycle ergometer exercise in COPD. Eur Respir J 2009;34(3):605-615.

33. Mahler DA, Gifford AH, Waterman LA, Ward J, Machala S, Baird JC' Mechanism of greater oxygen desaturation during walking compared with cycling in patients with COPD. Chest 2011;140(2):351-358.

34. Mathur RS, Revill SM, Vara DD, Walton R, Morgan MD. Comparison of peak oxygen consumption during cycle and treadmill exercise in severe chronic obstructive pulmonary disease. Thorax 1995; 50(8):829-833.

35. Spruit MA, Singh SJ, Garvey C, ZuWallack R, Nici L, Rochester C, et al. An official American Thoracic Society/European Respiratory Society statement: key concepts and advances in pulmonary rehabilitation. Am J Respir Crit Care Med 2013;188:e13-e64.

36. Gosselink R, Troosters T, Decramer M. Peripheral muscle weakness contributes to exercise limitation in COPD. Am J Respir Crit Care Med 1996;153(3):976-980.
37. McCarthy B, Casey D, Devane D, Murphy K, Murphy E, Lacasse Y. Pulmonary rehabilitation for chronic obstructive pulmonary disease (Review). Cochrane Database Syst Rev 2015;2:CD003793.

38. Márquez-Martín E, Ruiz FO, Ramos PC, López-Campos JL, Azcona BV, Cortés EB. Randomized trial of noninvasive ventilation combined with exercise training in patients with chronic hypercapnic failure due to chronic obstructive pulmonary disease. Respiratory Medicine 2014;108(12):1741-1751

39. Ferrazza AM, Martolini D, Valli G, Palange P. Cardiopulmonary exercise testing in the functional and prognostic evaluation of patients with pulmonary diseases. Respiration 2009;77(1):3-17.

40. Carvalho LA, Brandão DC, Campos SL, Vidal TMS, Remígio MI, Martins SM, Andrade AD. Noninvasive ventilation before maximum exercise test increases exercise tolerance in subjects with heart failure: a crossover study. Arch Phys Med Rehabil 2017; 98(5):849-855.

41. Borghi-Silva A, Mendes RG, Trimer R, Oliveira CR, Fregonezi GA, Resqueti VR, et al. Potential effect of 6 versus 12 weeks of physical training on cardiac autonomic function and exercise capacity in chronic obstructive pulmonary disease. Eur J Phys Rehabil Med 2015;51(2):211-221.

42. Olper L, Cervi P, De Santi F, Meloni C, Gatti R. Validation of the treadmill six-minute walk test in people following cardiac surgery. Phys Ther 2011;91:566-576.

43. Giardini A, Specchia S, Berton E, Sangiorgi D, Coutsoumbas G, Gargiulo G, et al. Strong and independent prognostic value of peak circulatory power in adults with congenital heart disease. Am Heart J 2007;154(3):441-447.

44. Tantucci C. Expiratory flow limitation definition, mechanisms, methods, and significance. Pulm Med 2013;2013:749860.

45. Kneidinger N, Gloeckl R Schönheit-Kenn U, Milger K, Hitzl W, Behr J, Kenn K. Impact of nocturnal noninvasive ventilation on pulmonary rehabilitation in patients with end-stage lung disease awaiting lung transplantation. Respiration 2018;95(3):161-168. 